\title{
Amylase-Protected Ag Nanodots for in vivo Fluorescence Imaging and Photodynamic Therapy of Tumors
}

This article was published in the following Dove Press journal: International Journal of Nanomedicine

\author{
Shuguang Wen ${ }^{1-3, *}$ \\ Weili Wang ${ }^{4, *}$ \\ Ruimin Liu $^{3}$ \\ Pengcheng $\mathrm{He}^{1}$
}

'Department of Hematology, The First Affiliated Hospital of Xi'an Jiaotong University, Xi'an 71006I, People's Republic of China; ${ }^{2}$ Department of Hematology, The First Affiliated Hospital of Zhengzhou University, Zhengzhou 450052, People's Republic of China; ${ }^{3}$ Basic Medical College, Henan University, Kaifeng 475000, People's Republic of China; ${ }^{4}$ State Key Laboratory of Radiation Medicine and Protection, School for Radiological and Interdisciplinary Sciences (RAD-X), Collaborative Innovation Center of Radiological Medicine of Jiangsu Higher Education Institutions, Soochow University, Suzhou 215123, People's Republic of China

*These authors contributed equally to this work
Correspondence: Pengcheng $\mathrm{He}$ Department of Hematology, The First Affiliated Hospital of Xi'an Jiaotong University, No. 277 Yanta West Road, Xi'an 7I006I, People's Republic of China Tel +86 I89 91232609

Email hepengcheng@xjtu.edu.cn
Background: Fluorescent metallic nanodots (NDs) have become a promising nanoprobe for a wide range of biomedical applications. Because Ag NDs have a high tendency to be oxidized, their synthesis and storage are a big challenge. Thus, the method for preparing stable Ag NDs is urgently needed. Surface modification and functionalization can enrich the capability of Ag NDs.

Methods: In this work, fluorescent Ag NDs were synthesized in deoxygenated water by using porcine pancreatic $\alpha$-amylase (PPA) as the stabilizing/capping agent. The absorption and fluorescence of PPA-protected Ag NDs (PPA@AgNDs) were measured with a spectrophotometer and a spectrofluorometer, respectively. The morphology of PPA@AgNDs was characterized by high-angle annular dark-field (HAADF) scanning transmission electron microscopy (STEM). The biocompatibility of PPA@AgNDs was evaluated by tetrazolium (MTT)-based assay. PolyLys-Cys-SH (sequence: KKKKKKC) peptides were conjugated to PPA@AgNDs via heterobifunctional crosslinkers. PolyLys-Cys-linked PPA@AgNDs absorbed 5-aminolevulinic acid (ALA) by electrostatic interaction at physiological $\mathrm{pH}$. The capability of tumor targeting was evaluated by intravenously injecting PPA@AgND-ALA into 4T1 breast cancer xenograft mouse models. Photodynamic therapy (PDT) against tumors was performed under $635 \mathrm{~nm}$ laser irradiation.

Results: PPA@AgNDs emitted at $640 \mathrm{~nm}$ with quantum yield of 2.1\%. The Ag NDs exhibited strong photostability over a long period and a fluorescence lifetime of $5.1 \mathrm{~ns}$. PPA@AgNDs easily entered the cells to stain the nuclei, showing the capabilities of living cell imaging with negligible cytotoxicity. ALA-loaded PPA@AgNDs (PPA@AgND-ALA) presented the superiority of passive tumor targeting via the enhanced permeability and retention (EPR) effect. Tumors were visualized in the near-infrared (NIR) region with reduced background noise. ALA molecules released from PPA@AgND-ALA was converted into the photosensitizer (PS) of protoporphyrin IX (PpIX) intracellularly and intratumorally, which greatly improved the PDT efficacy.

Conclusion: Our approach opens a new way to design a novel theranostic nanoplatform of PPA@AgND-ALA for effective tumor targeting and fluorescence image-guided PDT.

Keywords: $\alpha$-amylase, Ag nanodots, peptides, targeted imaging, photodynamic therapy

\section{Introduction}

Noble metal ( $\mathrm{Au}, \mathrm{Ag}, \mathrm{Cu}$ and $\mathrm{Pt}$ ) nanodots (NDs) have recently become a useful tool in diverse biomedical applications. ${ }^{1-11}$ These NDs exhibit discrete electronic structure, photoluminescence (PL), intrinsic chirality, catalytic activity, and paramagnetism. ${ }^{12-18}$ Moreover, the toxicity of NDs is much lower than heavy metal-containing fluorescent 
nanoprobes like quantum dots, making them as a promising alternative for biological imaging. ${ }^{2,4,8-11}$ So far, Ag NDs have not attracted as much attention as $\mathrm{Au}$ NDs due to their rapid decomposition to Ag ions upon exposure to water and oxygen, which is a big issue remaining unsolved. ${ }^{19}$ Unfortunately, the synthetic strategies of Au NDs are not all working for Ag NDs. For example, a few proteins such as bovine serum albumin (BSA), lysozyme, apo- $\alpha$-lactalbumin, horseradish peroxidase (HRP) and bovine pancreatic ribonuclease A (RNase A) were reported to synthesize Au NDs. ${ }^{20-24}$ The redox-active Tyr and Trp residues of proteins are too weak to reduce $\mathrm{Ag},{ }^{20}$ since the redox potential of $\mathrm{Ag}^{+} / \mathrm{Ag}$ is as low as $\sim 0.80 \mathrm{~V}$. Hence, a stronger reducing agent like $\mathrm{NaBH}_{4}$ is needed. Xie and coworkers were the first group to report the synthesis of $\mathrm{Au}$ NDs stabilized by BSA. ${ }^{20}$ Shortly afterwards, the method was extended by Guével et al to prepare Ag NDs based on the reduction by $\mathrm{NaBH}_{4}{ }^{25}$ However, the strong reducibility of $\mathrm{NaBH}_{4}$ made the reaction difficult to be controlled. Moreover, the protein coating increased the overall size. Likewise, $\mathrm{Yu}$ et al reported that fluorescent Ag NDs were synthesized within cellular nucleoli by photoactivation, depending on the high affinity of nucleolin to Ag ions. ${ }^{26}$

In this paper, we employ porcine pancreatic $\alpha$-amylase (PPA) as the stabilizing agent to synthesize fluorescent Ag NDs. As a single polypeptide chain, PPA (molecule weight: $51-54 \mathrm{kDa}$ ) is comprised of 475 residues to hydrolyze $\alpha$-bonds of $\alpha$-linked polysaccharides. ${ }^{27}$ It has two thiol groups and four disulfide bridges to offer a strong affinity for Ag. PPA-protected Ag NDs (PPA@AgNDs) exhibit strong red emission with peak maximum at $\sim 640$ $\mathrm{nm}$ and robust photostability, benefiting from our established method that can prevent Ag from tarnishing. The condense PPA coating isolates the Ag surface from corrosive $\mathrm{O}_{2}$ in water for high stability. Moreover, Ag NDs can be internalized by living cells and even enter the nuclei to show the capability of cell imaging with negligible cytotoxicity. To construct a multifunctional nanoplatform, positively charged lysine-rich polyLys-Cys-SH (sequence: KKKKKKC) peptides are covalently conjugated to $\mathrm{Ag}$ NDs by PEGylated SMCC crosslinker, and then negatively charged 5-aminolevulinic acid (ALA) is electrostatically adsorbed at physiological $\mathrm{pH}$ to form ALA-loaded PPA@AgNDs (PPA@AgND-ALA). Unlike many emerging therapeutic nanoplatforms, ${ }^{28-30}$ ALA has been clinically used as a prodrug to induce the tumor accumulation of protoporphyrin IX (PpIX) for photodynamic therapy (PDT). ${ }^{31-33}$ PPA@AgND-ALA can passively target tumors through the enhanced permeability and retention
(EPR) effect, and be endowed with fluorescent imageguided enhanced PDT of tumors.

\section{Methods}

\section{Chemicals}

Porcine pancreatic $\alpha$-amylase (PPA), 5-aminolevulinic acid (ALA), Rhodamine 6G (R6G), acetylacetone, formaldehyde and Dulbecco's Modified Eagle Medium (DMEM) were purchased from Sigma-Aldrich. SM (PEG) 12 crosslinkers and Slide-A-Lyzer ${ }^{\mathrm{TM}}$ Dialysis Cassettes (MWCO: $10 \mathrm{KDa}$ ) were purchased from Thermo Fisher Scientific Co., Ltd. Silver nitrate $\left(\mathrm{AgNO}_{3}\right)$, sodium hydroxide $(\mathrm{NaOH})$, sodium borohydride $\left(\mathrm{NaBH}_{4}\right)$ and dimethyl sulfoxide (DMSO) were purchased from Sinopharm Chemical Reagent Co., Ltd. All chemicals were used as received without further purification. All solutions were prepared using purified water with resistivity of $18 \mathrm{~m} \Omega \mathrm{cm}^{-1}$ as the solvent. The water was deoxygenated before use with a nitrogen purge for $1 \mathrm{~h}$ at the flow rate of $25 \mathrm{~mL} / \mathrm{s}$.

\section{Synthesis of PPA@AgNDs}

In a typical synthesis, $1 \mathrm{~mL}$ of $\mathrm{AgNO}_{3}$ solution $(10 \mathrm{mM})$ was mixed with $1 \mathrm{~mL}$ of PPA solution $(50 \mathrm{mg} / \mathrm{mL})$ under stirring in an ice bath for $1 \mathrm{~min}$. Thereafter, $0.1 \mathrm{~mL}$ of $\mathrm{NaOH}(1 \mathrm{M})$ was dropwise added into the mixture. 0.05 $\mathrm{mL}$ of $\mathrm{NaBH}_{4}$ solution $(2 \mathrm{mM})$ was added. The color of solution changed from colorless to light brown and then to reddish brown, which indicated the formation of Ag NDs. The resultant solution was dialyzed with a dialysis cassette (MWCO: $10 \mathrm{KDa}$ ) in PBS solution (pH 7.4) and kept at $4{ }^{\circ} \mathrm{C}$ in darkness for the follow-up use.

\section{Conjugation of PolyLys-Cys-SH (KKKKKKC) to PPA@AgNDs}

Two milligrams of polyLys-Cys-SH peptides were dissolved in $20 \mu \mathrm{L}$ of DMSO and mixed with $25 \mathrm{mg} / \mathrm{mL}$ PPA@AgNDs in $1 \mathrm{~mL}$ of PBS solution ( $\mathrm{pH} 7.2)$. Then, SM (PEG) 12 crosslinkers with $1 \mathrm{mM}$ final concentration were added and stirred for $2 \mathrm{~h}$ at $4^{\circ} \mathrm{C}$. The NHS esters of SM (PEG) 12 reacted with the primary amines of PPA to form amide bonds, while the maleimides of SM $(\mathrm{PEG})_{12}$ reacted with the sulfhydryl groups of polyLysCys-SH peptides to form thioether bonds. Thus, polyLys-Cys-SH peptides were covalently conjugated to PPA@AgNDs. The resultant solution was dialyzed for $1 \mathrm{~h}$ with a dialysis cassette in PBS solution $(\mathrm{pH}$ 
7.4). Peptide-linked PPA@AgNDs was purified and kept at $4{ }^{\circ} \mathrm{C}$ in darkness.

\section{Electrostatic Adsorption of 5-Aminolevulinic Acid (ALA)}

Conjugation of ALA to PPA@AgNDs modified with polyLys-Cys-SH peptides was achieved by electrostatic interaction between ALA and the positively charged lysine residues. Four milligrams of ALA was dissolved in $2 \mathrm{~mL}$ of Dulbecco's Modified Eagle Medium (DMEM) and sterilized with filtration through a $0.2 \mu \mathrm{m}$ filter. PolyLys-Cyslinked PPA@AgNDs were also sterilized. After that, $1 \mathrm{~mL}$ of ALA solution was mixed with $200 \mu \mathrm{L}$ of polyLys-Cyslinked PPA@AgNDs, and the system pH was adjusted to pH 7.4 with $1 \mathrm{~N} \mathrm{NaOH}$ solution. The resultant solution was dialyzed for $1 \mathrm{~h}$ with a dialysis cassette (MWCO: 10 $\mathrm{KDa}$ ) in PBS solution ( $\mathrm{pH}$ 7.4) to remove unbound ALA. After the synthesis of PPA@AgND-ALA, the unbound ALA was collected and its concentration in solvent was determined using a method reported by AI-Kassas et al. ${ }^{34}$ ALA loading was calculated from:

ALA loading efficiency

$$
\begin{aligned}
& =\frac{\text { mass of total ALA }- \text { mass of ALA in solvent }}{\text { mass of total ALA }} \times 100 \% \\
& =21.4 \%
\end{aligned}
$$

\section{Materials Characterizations}

Fluorescence spectra were measured with a Hitachi F-4600 fluorometer. UV-visible absorption spectra were recorded with a Shimadzu UV-2600 spectrophotometer. Ambientlight and dark-field photographs of PPA@AgNDs were captured with an Olympus Stylus 7010 digital camera. Time-resolved fluorescence measurement was carried out with an FLS 920 fluorescence lifetime and steady-state spectroscopy. Scanning transmission electron microscopy (STEM) was recorded using a TEM/STEM microscope with an FEI Tecnai G2 F20 field emission gun and fitted with a Fischione HAADF detector, an $80 \mathrm{~mm}^{2}$ Oxford Instruments X-Max silicon drift detector (SDD) and a Gatan Orius. TEM sample was prepared by drop-casting PPA@AgNDs on copper grids coated with ultrathin carbon film and dried at ambient temperature and pressure conditions. Dynamic light scattering (DLS) and zeta potential (ZP) were carried out with a Mastersizer 3000 laser diffraction particle size analyzer.

\section{Calculation of Quantum Yield}

The quantum yield $(\Phi)$ of PPA@AgNDs is determined by the relative method and calculated according to the following equation:

$$
\Phi=\Phi_{R}\left(\frac{I}{I_{R}}\right)\left(\frac{A_{R}}{A}\right)
$$

where $\Phi_{R}$ is the quantum yield of the reference R6G $\left(\Phi_{R}=94 \%\right) ; \mathrm{I}$ and $\mathrm{I}_{\mathrm{R}}$ are the integrated fluorescence intensities for PPA@AgNDs and R6G, respectively; A and $A_{R}$ are the absorbance values of PPA@AgNDs and R6G, respectively.

\section{Cell Viability Assay}

Mouse breast (4T1) cancer cells were cultured as adherent monolayers in plastic tissue culture dishes in RPMI-1640 medium (ATCC, USA) supplemented with 10\% heatinactivated Fetal Bovine Serum (ATCC, USA) and antibiotic solution ( $1 \mathrm{~mL}$ per $100 \mathrm{~mL}$ of cell culture medium, Thermo Fisher Scientific, USA). The cells were cultured in $75 \mathrm{~cm}^{2}$ culture flasks and maintained within a Thermo Scientific Forma Direct-Heat $\mathrm{CO}_{2}$ incubator $\left(37^{\circ} \mathrm{C}, 5 \%\right.$ $\mathrm{v} / \mathrm{v} \mathrm{CO}_{2}$ atmosphere and $95 \%$ relative humidity). The cytotoxicity of PPA@AgNDs on 4T1 cells was assessed by MTT (3(4,5-dimethylthiazol-2-yl) 2,5-diphenyltetrazolium bromide) assay. Cells were seeded into a 96-well plate at the density of $1.0 \times 10^{4}$ cells/well in $100 \mu \mathrm{L}$ of complete growth medium. After overnight initial plating, the medium was exchanged for fresh medium supplemented with PPA@AgNDs within a range from 0, 10, 100 to $200 \mu \mathrm{g} / \mathrm{mL}$ for $48 \mathrm{~h}$ with three wells per condition. PPA@AgNDs were dissolved in $1 \times$ PBS, and the final concentration was obtained by diluting PPA@AgNDs with complete culture medium. Fresh medium was used as untreated control group. Twenty microliters of MTT working solution $(5 \mathrm{mg} / \mathrm{mL})$ was added. After 4 hof incubation at $37^{\circ} \mathrm{C}$, culture supernatant was removed from the wells, and the formazan crystals were dissolved in $150 \mu \mathrm{L}$ of DMSO. One hundred microliters of the supernatant was transferred into a new 96-well plate after centrifugation (1000 g, $5 \mathrm{~min}$ ). The samples were analyzed with an EL $\times 800$ absorbance microplate reader (BioTek Instruments, Winooski, USA) by measuring $570 \mathrm{~nm}$ reading with background correction at $630 \mathrm{~nm}$. Healthy cells were used as control, so that the cell viability is regarded as $100 \%$. The cell viability of PPA@AgND-treated cells was indicated as the percentage (\%) of the control. 


\section{Apoptosis Analysis}

4T1 cells were plated in a 24 -well plate $\left(1 \times 10^{5}\right.$ cells/well $)$ and incubated with gradient concentrations $(0,10,100$, and $200 \mu \mathrm{g} / \mathrm{mL}$ ) of PPA@AgNDs for $48 \mathrm{~h}$ at $37^{\circ} \mathrm{C}$. The cells were gathered and washed twice with PBS, and resuspended in $200 \mu \mathrm{L}$ of binding buffer and incubated with $5 \mu \mathrm{L}$ of annexin V-FITC and $1 \mu \mathrm{L}$ of PI at room temperature, hatched at room temperature (RT) for $20 \mathrm{~min}$ in the dark. Then, $400 \mu \mathrm{L}$ of binding buffer was added, gently mixed, put over the ice and immediately analyzed using a fluorescence-activated cell sorting (FACS) system (FC500 flow cytometer).

\section{Tumor-Targeted Imaging in vitro and in vivo}

In vitro cell imaging: Cell culture medium was changed daily. The cell medium was replaced by the fresh on the day of the test. After 2 h of incubation with PPA@AgNDs, the cells were washed thrice with $1 \times \mathrm{PBS}$ buffer to wipe out unbound PPA@AgNDs.PPA@AgND-staining cells were under microscopic observation with a Leica SP5 confocal microscope.

In vivo tumor-targeted imaging: $100 \mu \mathrm{L}$ of PPA@AgNDALA was intravenously (IV) injected into 4T1 tumor xenografted nude mice models $(\mathrm{n}=3)$. The time-course in vivo and ex vivo imaging was carried out with an IVIS $^{\circledR}$ Spectrum in vivo imaging system.

\section{PDT in vitro and in vivo}

In vitro PDT: $4 \mathrm{~T} 1$ cells $\left(1 \times 10^{4}\right.$ cells $\left./ 100 \mu \mathrm{L}\right)$ were seeded in 96-well plates and placed in the incubator at $37^{\circ} \mathrm{C}$ for 24 $\mathrm{h}$. The medium was then replaced with fresh one containing $100 \mu$ M PPA@AgNDs or PPA@AgND-ALA. After 6 h, the medium was replaced again with $1 \times$ PBS. Cells were illuminated under a $635-\mathrm{nm}$ laser at the fluence rate of $100 \mathrm{~mW} / \mathrm{cm}^{2}$ for $10 \mathrm{~min}$. Immediately afterwards, PBS was replaced again with fresh medium. However, the control group was not exposed to ALA or laser irradiation. After $24 \mathrm{~h}$, cell viability was tested using MTT assay. The absorbance of samples was measured with the $\mathrm{EL} \times 800$ microplate reader by measuring $570 \mathrm{~nm}$ reading with background correction at $630 \mathrm{~nm}$.

In vivo PDT: 12 nude mice bearing 4T1 tumors were randomly distributed into four groups: (1) control group, injection of PBS $(100 \mu \mathrm{L})$; (2) "laser" group, $635 \mathrm{~nm}$-laser irradiation (0.5 W/cm² $10 \mathrm{~min})$; (3) "PPA@AgND-ALA" group, injection of PPA@AgND-ALA $(100 \mu \mathrm{L}, 100 \mu \mathrm{g} /$ $\mathrm{mL})$; (4) "PPA@AgND-ALA + laser" group, injection of PPA@AgND-ALA $(100 \mu \mathrm{L}, 100 \mu \mathrm{g} / \mathrm{mL})$ with $635 \mathrm{~nm}$ laser irradiation $\left(0.5 \mathrm{~W} / \mathrm{cm}^{2}, 10 \mathrm{~min}\right)$. For group (4), PDT was carried out on the tumor site at $4 \mathrm{~h}$ post-injection. Tumor volume and body weight were recorded every 4 days over a 24-day period. After the treatment, major organs (heart, liver, spleen, lung and kidney) and tumors of mice were collected for the hematoxylin and eosin (H\&E) stain.

\section{Results and Discussion Synthesis of PPA@AgNDs}

Figure 1 describes the synthesis procedures of fluorescent Ag NDs based on the kinetic template effect of PPA. Firstly, $\mathrm{AgNO}_{3}$ was mixed with PPA in aqueous solution under vigorous stirring, and the metal cations were coordinated to cysteine (Cys) residues. Secondly, $\mathrm{NaBH}_{4}$ was added to reduce the $\mathrm{Ag}$ ions from +1 to 0 . Four disulfide bonds (S-S) broke into eight thiols to stabilize the Ag NDs with two extra thiols. $\mathrm{NaBH}_{4}$ played a vital role in the synthesis of $\mathrm{Ag} \mathrm{NDs}$, because too much $\mathrm{NaBH}_{4}$ resulted in large, non-fluorescent Ag NPs. Thus, the synthesis has to be progressed in low temperature to decelerate the redox reaction. As the solvent, water was deoxygenated beforehand with a nitrogen purge for $1 \mathrm{~h}$ at the flow rate of $25 \mathrm{~mL} / \mathrm{s}$ to minimize dissolved oxygen, so PPA@AgNDs were less likely to be oxidized throughout manipulation. PPA@AgNDs were purified by dialysis in $1 \times$ PBS and stored at $4{ }^{\circ} \mathrm{C}$ in darkness for subsequent use.
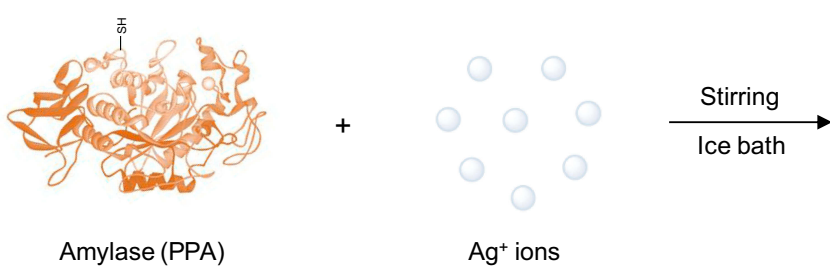

Figure I Schematic of synthesis of fluorescent PPA@AgNDs.
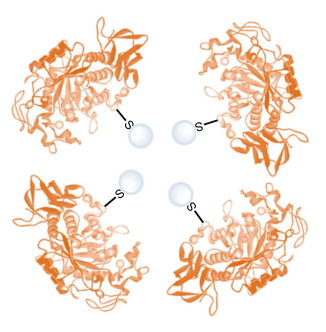

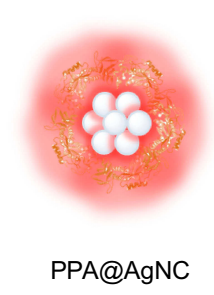




\section{Photophysical Properties of PPA@AgNDs}

As shown in Figure 2A, the UV/vis absorption of PPA@AgNDs has a small shoulder peak at 420 nm because of surface plasma resonance (SPR) absorption. Figure 2B presents that nearly a half of the band falls in the red spectrum (580 to $700 \mathrm{~nm}$ ) with a peak maximum at $643 \mathrm{~nm}$ and a narrow full width at half maximum (FWHM) of $\sim 126 \mathrm{~nm}$. The quantum yield (QY) of PPA@AgNDs was determined as $2.1 \%$ using Rhodamine 6G (R6G) in ethanol as the reference standard $(\mathrm{QY}=94 \%)$. As shown in Figure $2 \mathrm{C}$, the strong photostability is noted that the fluorescence intensity of PPA@AgNDs deceased slowly ( $<10 \%$ ) over a 30-day storage, confirming that the oxidation process was inhibited to a great extent. Although $\mathrm{O}_{2}$ gas had been largely removed from water by $\mathrm{N}_{2}$ gas bubbling, the air could still dissolve in water over
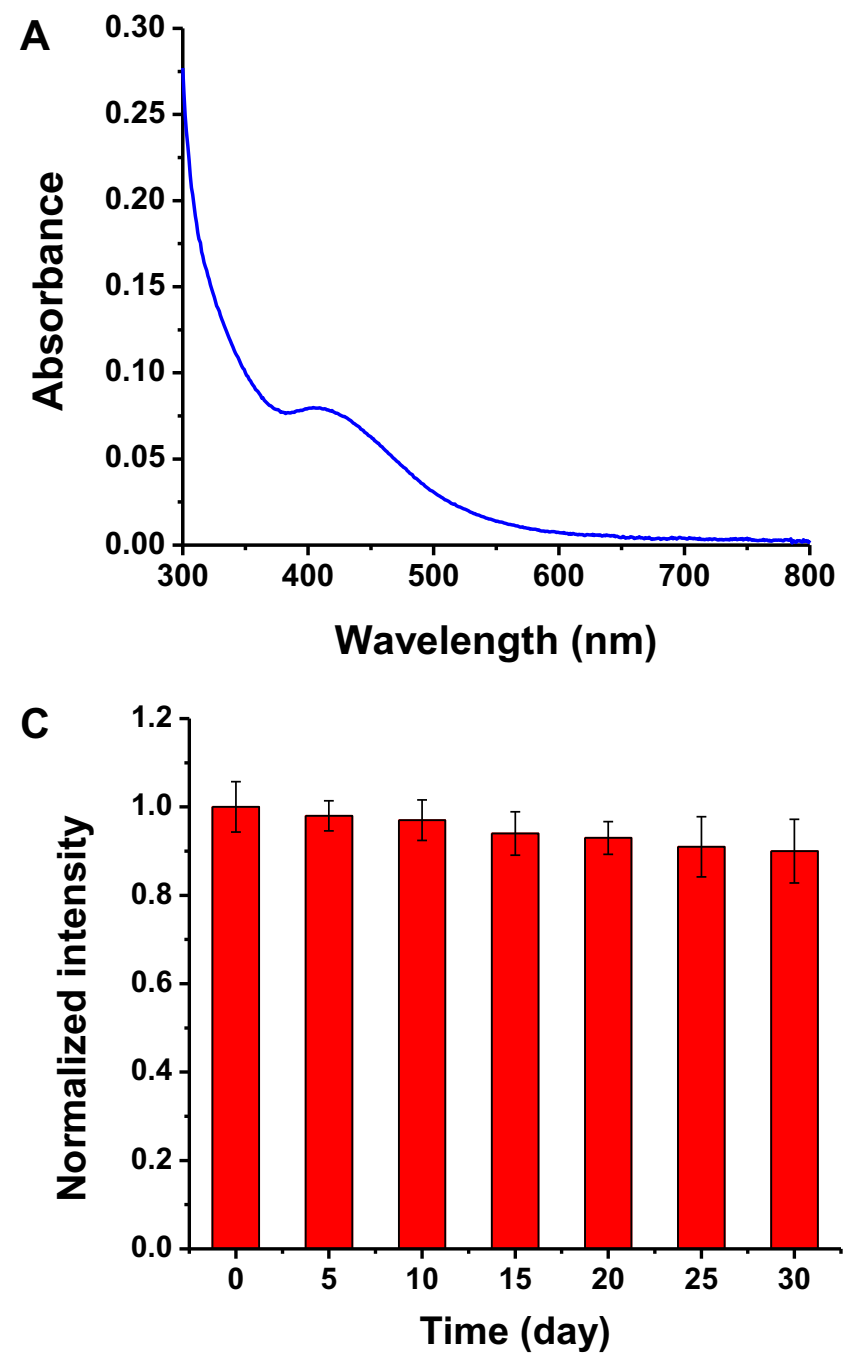

time. However, the re-dissolved $\mathrm{O}_{2}$ had little effect on the fluorescence intensity of PPA@AgNDs, indicating that PPA might form a condense protein coating to isolate the Ag NDs from the environment. Changes in $\mathrm{pH}$ also had unobvious effect on the intensity (Figure 2D). All the advantages of PPA@AgNDs are favorable for in vivo fluorescence imaging. As shown in Figure S1, the time-resolved fluorescence decay curve is well fitted using a three-exponential decay function with an average lifetime (T) of $5.12 \mathrm{~ns}(\mathrm{~T} 1=4.69 \mathrm{~ns}, 45.47 \%$; $\mathrm{T} 2=1.21 \mathrm{~ns}, 7.48 \%$; T3 = $12.68 \mathrm{~ns}, 47.05 \%$ ).

\section{Morphology and Size Analysis of PPA@AgNDs}

High-angle annular dark-field (HAADF) scanning transmission electron microscopy (STEM) is advantageous for morphological analysis of metallic NDs. The image contrast of
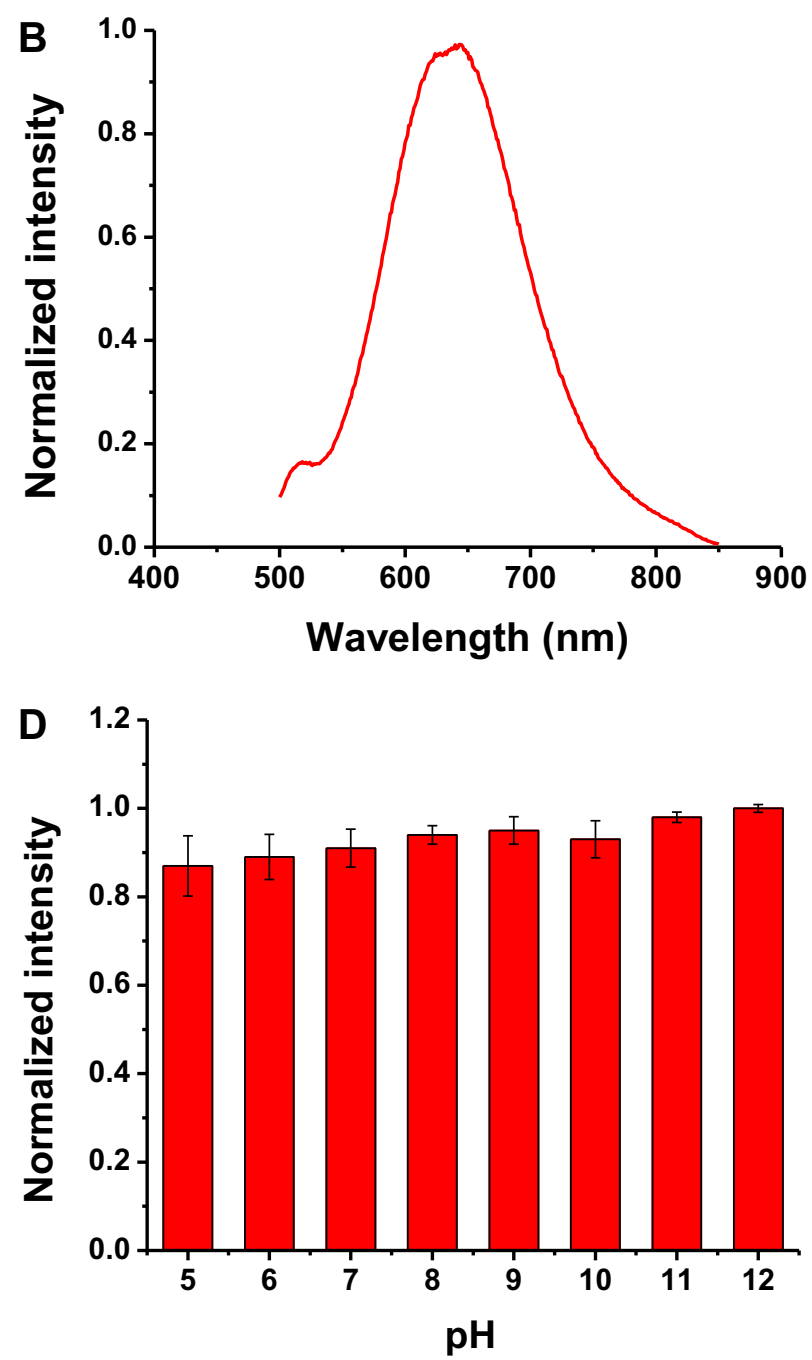

Figure 2 (A) Typical UV-vis absorption and (B) normalized fluorescence spectra of PPA@AgNDs in aqueous solution. (C) Effect of air oxidation on fluorescence intensity of PPA@AgNDs over a long storage (30 days). (D) Effect of pH on fluorescence intensity of PPA@AgNDs $\left(\lambda_{\text {ex }}: 490\right.$ nm). 
Ag NDs is proportional to the atomic number $(Z)$ squared of Ag element. As shown in Figure 3A, the white dots represent the Ag NDs, and the average diameter is $1.72 \pm 0.29 \mathrm{~nm}$ (Figure 3 inset). The dynamic light scattering (DLS) spectrum of Figure 3B presents that the hydrodynamic diameter of PPA@AgNDs is determined to be 6-7 nm, indicating that the protein contributed little to size increase. As shown in Figure 3C, the matrix-assisted laser desorption/ionizationtime of flight (MALDI-TOF) mass spectrometry (MS) spectrum confirms that PPA@AgNDs have multiple species of different molecular weights, but the majority of them are composed of $19 \mathrm{Ag}$ atoms. It is worth mentioning that the Ag NDs were likely detached from the PPA coating during the MS measurement probably due to the bond breaking by the laser.

\section{Cell Imaging with PPA@AgNDs and PDT with PPA@AgND-ALA}

The ultrasmall size, good photostability and bright emission of PPA@AgNDs are favorable for sensitive cell imaging. As shown in the confocal laser scanning microscopy (CLSM) images of Figure 4A, 4T1 cells exhibit bright red emission after 2 h of incubation with PPA@AgNDs. The nuclei gave red fluorescent signals overlapping with the signal of 4',6-diamidino-2-phenylindole (DAPI) that stains the nuclei only. The result indicates that PPA@AgNDs passed through lysosomal and endosomal membrane to enter the nuclei without nucleitargeting agents. Unlikely, many other NPs tend to end up in lysosomes and endosomes after cellular uptake. Thus, further investigations are needed to explain this behavior. Flow cytometric assay was used for viability assessment of the cells treated with various concentrations $(0,10,100$ and 200 $\mu \mathrm{g} / \mathrm{mL}$ ) of PPA@AgNDs. As shown in Figure 4B, more than $90 \%$ of the cells are alive while apoptotic cells were less than 5\% as exposing to 10 or $100 \mu \mathrm{g} / \mathrm{mL}$ PPA@AgNDs. Although the FACS data confirm that the high concentration (such as 200 $\mu \mathrm{g} / \mathrm{mL})$ of PPA@AgNDs resulted in slightly cytotoxic effect (Figure 4C), they were biosafe over the abroad range of up to $100 \mu \mathrm{g} / \mathrm{mL}$. To synthesize a theranostic nanoplatform, positively charged polyLys-Cys-SH (KKKKKKC) peptides were conjugated to the PPA coating via covalent coupling between amine $\left(-\mathrm{NH}_{2}\right)$ and sulfhydryl (-SH) groups using a heterobifunctional crosslinker of $\mathrm{SM}(\mathrm{PEG})_{12}$ (Figure 5A). As the precursor of PpIX, negatively charged ALA was absorbed to the peptides by electrostatic adsorption to construct ALAloaded PPA@AgNDs (PPA@AgND-ALA), the hydrodynamic diameter of which reached $\sim 21 \mathrm{~nm}$ (Figure 5B). However, ALA can be released when the surrounding $\mathrm{pH}$ reduces to $\mathrm{pH} 5$ such as endosomal or lysosomal interior (Figure 5C). As shown in Figure 5D, the large portion $(\sim 70 \%)$ of cells are killed under 635-nm laser irradiation with the power density of $100 \mathrm{~mW} / \mathrm{cm}^{2}$ for $10 \mathrm{~min}$ in the presence of PPA@AgND-ALA. However, the laser or PPA@AgNDs alone was incapable of killing the cells.

\section{Tumor-Targeted Imaging and PDT with PPA@AgND-ALA}

As depicted in Figure 2B, a large portion of emission spectrum of PPA@AgNDs falls in the NIR region $(\lambda>700 \mathrm{~nm})$, which suggests that they are suitable for fluorescence imaging to
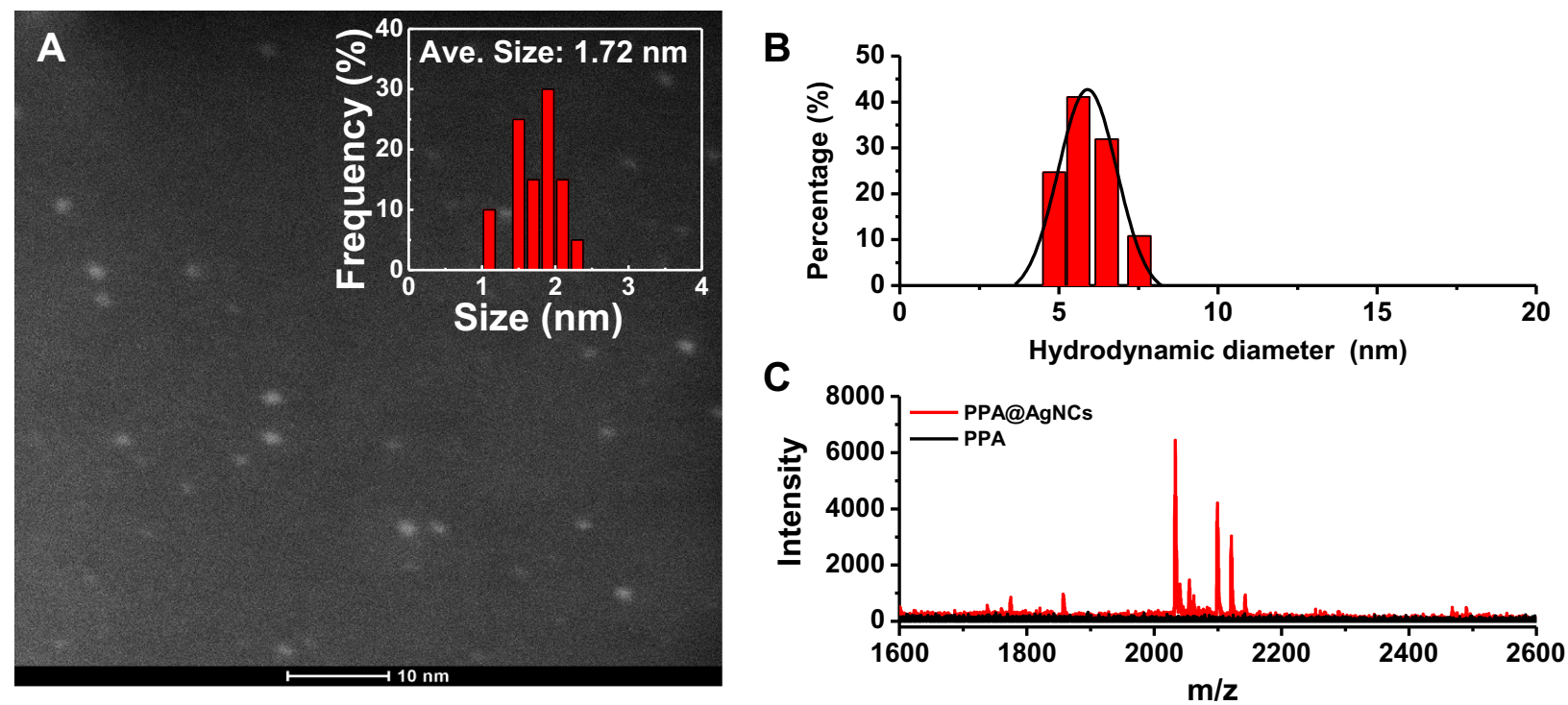

Figure 3 (A) HAADF-STEM image and particle size analysis. (B) DLS spectrum. (C) MALDI-TOF MS spectrum of PPA@AgNDs. 
A
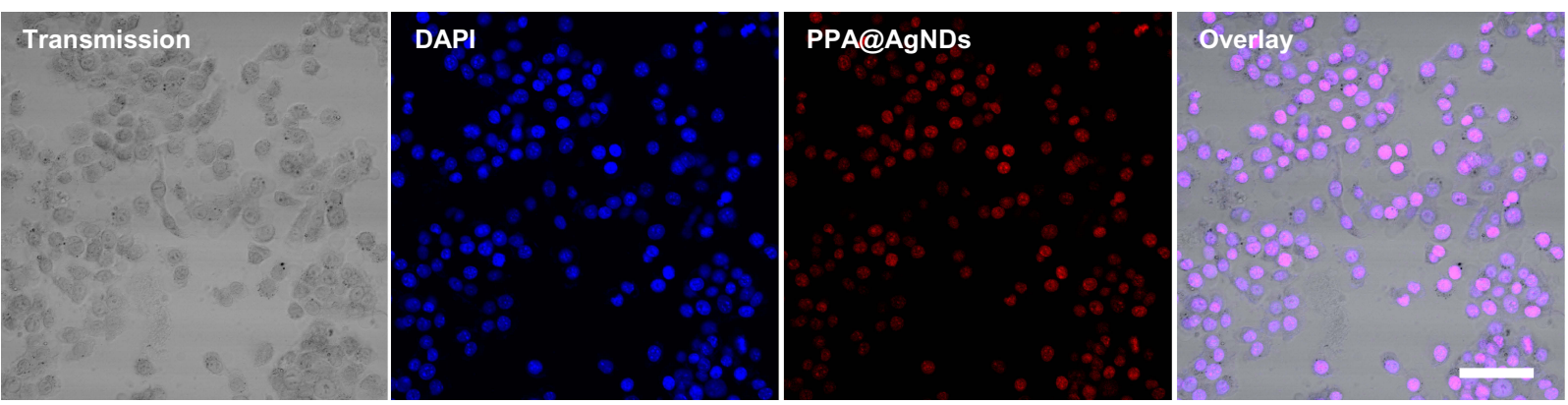

B

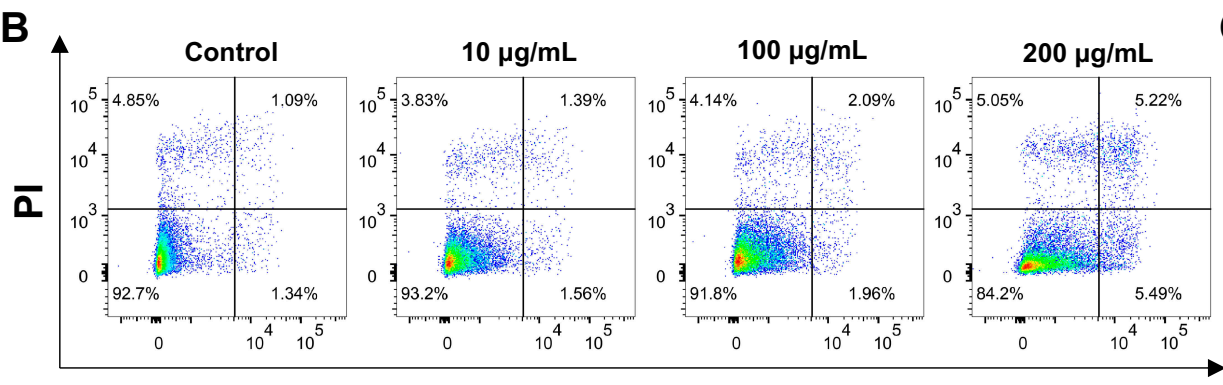

\section{Annexin V FITC}

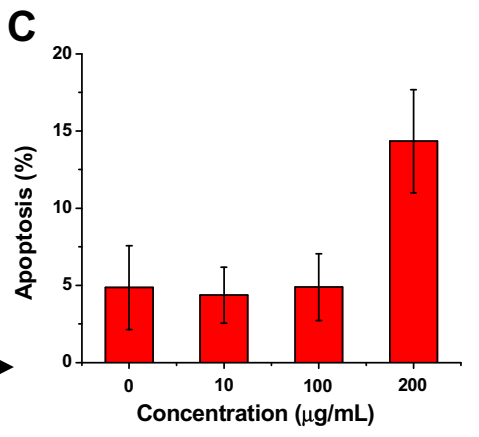

Figure 4 (A) Confocal laser scanning microscopy (CLSM) images of 4TI breast cancer cells incubated with PPA@AgNDs for 2 h. Scale bar: 200 nm. (B) Flow cytometric analysis and (C) apoptosis percentage of 4TI cells after incubating with 0, 10, 100 and $200 \mu \mathrm{g} / \mathrm{mL}$ of PPA@AgNDs.

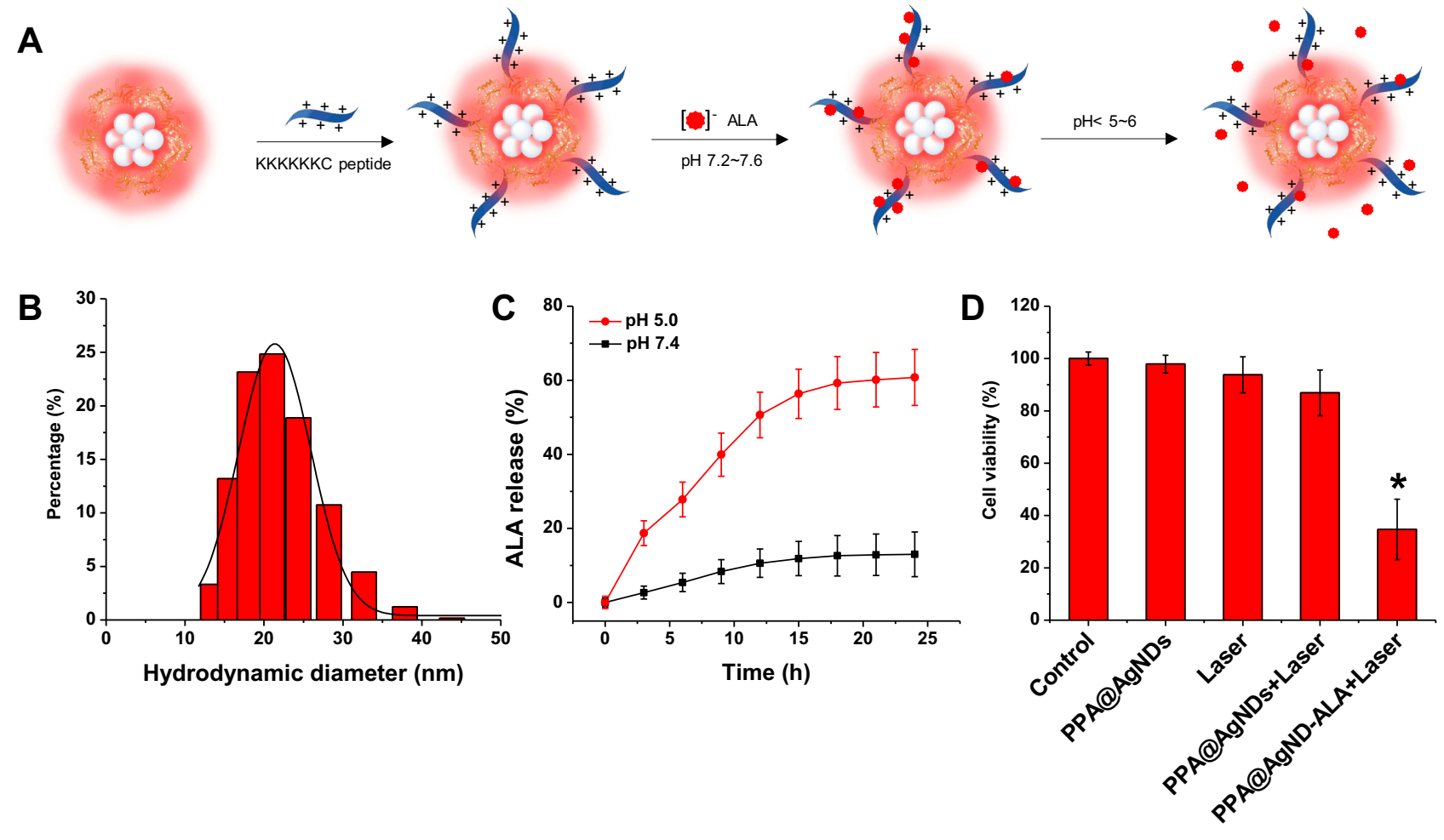

Figure 5 (A) Schematic of conjugation of polyLys-Cys-SH peptide to PPA@AgNDs at neutral pH; ALA loading to and releasing from PPA@AgND-ALA at acidic pH. (B) DLS spectrum of PPA@AgND-ALA. (C) Cumulative amount of ALA released from PPA@AgND-ALA at pH 5.0 and pH 7.4. (D) Cell viability of different treatment groups $(* p<0.05)$.

promote the signal-to-noise ratio (SNR). Indeed, the mouse shows fairly low autofluorescence background at pre-injection (Figure 6A). For in vivo fluorescence imaging, $200 \mu \mathrm{L}$ of
PPA@AgND-ALA (100 $\mu \mathrm{g} / \mathrm{mL})$ was intravenously injected into a mouse bearing 4T1 tumor xenograft. Figure 6A shows that PPA@AgND-ALA starts to accumulate in the tumor area 


\section{A}

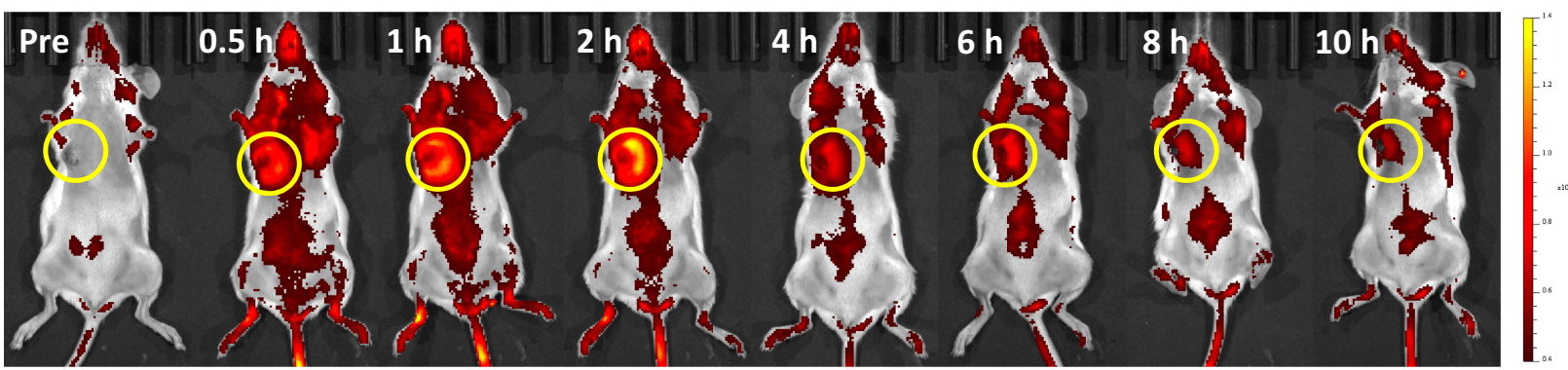

B

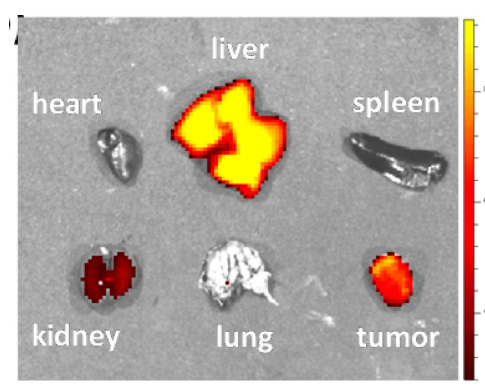

$\mathrm{C}^{10}$

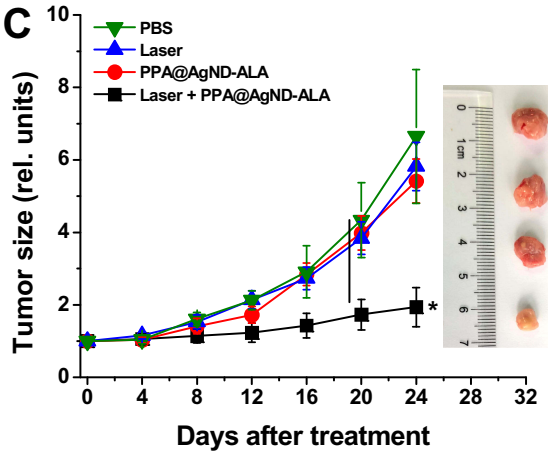

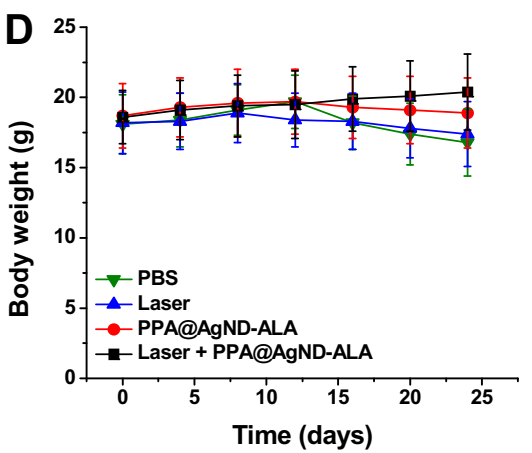
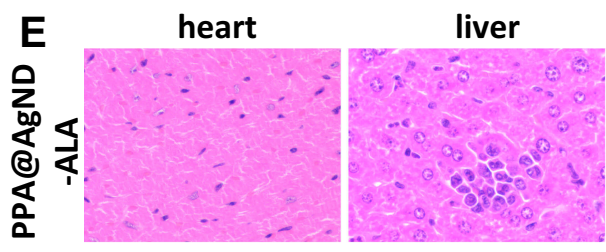

spleen

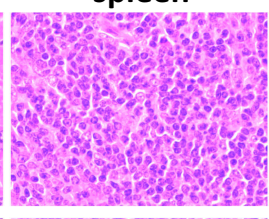

kidney
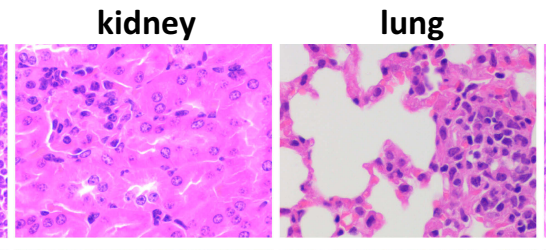

tumor

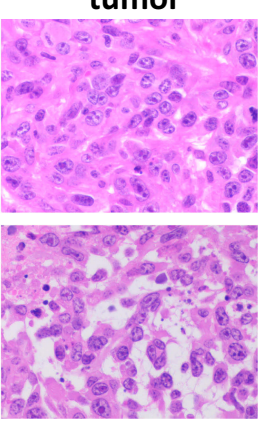

Figure 6 (A) In vivo time-course fluorescence images of a 4TI tumor xenografted mouse at pre-injection, 0.5, I, 2, 4, 6, 8 and I0 h post-injection of PPA@AgND-ALA. (B) Ex vivo fluorescence images of major organs (heart, liver, spleen, kidney, and lung) and the tumor collected at $10 \mathrm{~h}$ post-injection. (C) Tumor volume and (D) body weight of mice in various treatment groups over the experiment period. Data are presented in mean $\pm S D$ ( $n=3$ in each group). The Student's $t$-test is carried out to compare the intensity of different organs $(* p<0.05)$. (E) Histological H\&E staining of major organs and tumors, magnification: $\times 400$.

as quickly as $0.5 \mathrm{~h}$ post-injection (p.i.), where fluorescent signals gradually become strong. The tumor area exhibited the highest SNR value at $2 \mathrm{~h}$ p.i., but afterwards the intensity became weaker. The results definitely indicate that PPA@AgND-ALA was capable of passive tumor targeting via the enhanced permeability and retention (EPR) effect. At $10 \mathrm{~h}$ p.i., the mouse was sacrificed to collect its major organs including heart, spleen, liver, kidney, lung and tumor for ex vivo imaging (Figure 6B). As observed, the tumor gives very strong signals, while a large number of PPA@AgND-ALA retained in the reticuloendothelial system (RES) such as kidney and liver. The in vitro tests confirmed that PPA@AgNDALA actively killed 4T1 cells under laser irradiation, but the in vivo anti-tumor ability was needed to be tested. Hence, PPA@AgND-ALA was intravenously injected into the tumor- bearing mice. As shown in Figure 6C, the tumors of mice treated with PBS, laser or PPA@AgND-ALA show a similar trend to grow very rapidly, which rules out their individual role in tumor inhibition. In the group of mice injected with PPA@AgND-ALA followed by 635 nm laser irradiation (100 $\mathrm{mW} / \mathrm{cm}^{2}, 10 \mathrm{~min}$ ), the combinational use showed significant inhibitory effect on tumors. Their tumor sizes were much smaller than that of the control and other treatment groups, and no recrudescence was noticed. The results suggested that the ALA molecules intratumorally released from PPA@AgND-ALA strengthened the in vivo PDT efficacy. Figure $6 \mathrm{D}$ shows that the mice have a steady weight growth for "PPA@AgND-ALA + laser" group, but no obvious change for "laser" group. For the control group, the mouse weight had a slight decrease. As observed in the hematoxylin 
and eosin (H\&E) analysis (Figure 6E), major organs do not show distinct injury or inflammation. However, it was encouragingly observed that the damaged cells of the tumor after PDT treatment presented morphological characteristics greatly different from the control, and the majority of them were fragmented and disassembled.

\section{Conclusion}

To sum up, we successfully prepared fluorescent Ag NDs in water using PPA as the capping/stabilizing agent. PPA@AgNDs emitted at $\sim 640 \mathrm{~nm}$ with the QY of $2.1 \%$ and an average lifetime of $\sim 5.1 \mathrm{~ns}$. The attractive properties combining with ultrasmall size favored PPA@AgNDs to work well for both in vitro and in vivo fluorescence imaging. Moreover, PPA@AgNDs were biosafe and showed no cytotoxicity even at a high dosage. The positively charged polyLys-Cys-SH peptides were linked to PPA@AgNDs for electrostatic adsorption of ALA to synthesize PPA@AgND-ALA. Without further functionalization, PPA@AgND-ALA efficiently accumulated in the tumors depending on passive targeting via the EPR effect. Under $635 \mathrm{~nm}$ laser irradiation, ALA released from PPA@AgND promoted the PDT efficacy against tumors. All the results proved that PPA@AgND-ALA was a powerful nanoplatform for tumor-targeted PDT.

\section{Ethics Statement}

The experiments are conducted according to the Guide for the Care and Use of Laboratory Animals published by $\mathrm{NIH}$ and approved by the Animal Ethics Committee, School of Medicine, Xi'an Jiaotong University, China.

\section{Acknowledgments}

This work was financially supported by Key Research and Development Projects in Shaanxi Province of China (2018ZDCXL-SF-01-02-01).

\section{Disclosure}

The authors report no conflicts of interest in this work.

\section{References}

1. Jin R. Quantum sized, thiolate-protected gold nanoclusters. Nanoscale. 2010;2(3):343-362. doi:10.1039/b9nr00160c

2. Lin CA, Yang TY, Lee CH, et al. Synthesis, characterization, and bioconjugation of fluorescent gold nanoclusters toward biological labeling applications. ACS Nano. 2009;3(2):395-401. doi:10.1021/ nn800632j

3. Zheng J, Zhang C, Dickson RM. Highly fluorescent, water-soluble, size-tunable gold quantum dots. Phys Rev Lett. 2004;93(7):077402. doi:10.1103/PhysRevLett.93.077402
4. Zheng Y, Lai L, Liu W, Jiang H, Wang X. Recent advances in biomedical applications of fluorescent gold nanoclusters. $A d v$ Colloid Interface Sci. 2017;242:1-6. doi:10.1016/j.cis.2017.02.005

5. Choi S, Dickson RM, Yu J. Developing luminescent silver nanodots for biological applications. Chem Soc Rev. 2012;41(5):1867-1891. doi:10.1039/C1CS15226B

6. Díez I, Ras RH. Fluorescent silver nanoclusters. Nanoscale. 2011;3 (5):1963-1970. doi:10.1039/c1nr00006c

7. Petty JT, Story SP, Hsiang JC, Dickson RM. DNA-templated molecular silver fluorophores. J Phys Chem Lett. 2013;4(7):1148-1155. doi:10.1021/jz4000142

8. Das NK, Ghosh S, Priya A, Datta S, Mukherjee S. Luminescent copper nanoclusters as a specific cell-imaging probe and a selective metal ion sensor. $J$ Phys Chem C. 2015;119(43):24657-24664. doi:10.1021/acs.jpcc.5b08123

9. Wang C, Wang C, Xu L, Cheng H, Lin Q, Zhang C. Protein-directed synthesis of $\mathrm{pH}$-responsive red fluorescent copper nanoclusters and their applications in cellular imaging and catalysis. Nanoscale. 2014;6(3):1775-1781. doi:10.1039/C3NR04835G

10. Jia X, Li J, Han L, Ren J, Yang X, Wang E. DNA-hosted copper nanodots for fluorescent identification of single nucleotide polymorphisms. ACS Nano. 2012;6(4):3311-3317.

11. Tanaka S, Miyazaki J, Tiwari DK, Jin T, Inouye Y. Fluorescent platinum nanodots: synthesis, purification, characterization, and application to bioimaging. Angew Chem Int Ed. 2010;50:431-435. doi:10.1002/anie. 201004907

12. Aikens CM. Electronic structure of ligand-passivated gold and silver nanodots. J Phys Chem Lett. 2001;2:99-104. doi:10.1021/jz101499g

13. Wilcoxon JP, Martin JE. Photoluminescence from nanosize gold clusters. J Chem Phys. 1998;108:9137. doi:10.1063/1.476360

14 . Wu Z, Jin R. On the ligand's role in the fluorescence of gold nanoclusters. Nano Lett. 2010;10(7):2568-2573. doi:10.1021/nl101225f

15. Knoppe S, Bürgi T. Chirality in thiolate-protected gold clusters. Acc Chem Res. 2014;47(4):1318-1326. doi:10.1021/ar400295d

16. Li G, Jin R. Atomically precise gold nanoclusters as new model catalysts. Acc Chem Res. 2013;46(8):1749-1758. doi:10.1021/ar300213z

17. Tsunoyama H, Sakurai H, Negishi Y, Tsukuda T. Size-specific catalytic activity of polymer-stabilized gold nanodots for aerobic alcohol oxidation in water. $J$ Am Chem Soc. 2005;127(26):9374-9375. doi:10.1021/ja052161e

18. Santiago González BS, Rodríguez MJ, Blanco C, Rivas J, Lópezquintela MA, Martinho JMG. One step synthesis of the smallest photoluminescent and paramagnetic PVP-protected gold atomic clusters. Nano Lett. 2010;10(10):4217-4221. doi:10.1021/nl1026716

19. Sachan R, Ramos V, Malasi A, et al. Oxidation-resistant silver nanostructures for ultrastable plasmonic applications. Adv Mater. 2013;25(14):2045-2050. doi:10.1002/adma.201204920

20. Xie J, Zheng Y, Ying JY. Protein-directed synthesis of highly fluorescent gold nanoclusters. J Am Chem Soc. 2009;131(3):888-889. doi:10.1021/ja806804u

21. Russell BA, Jachimska B, Komorek P, Mulheran PA, Chen Y. Lysozyme encapsulated gold nanodots: effects of cluster synthesis on natural protein characteristics. Phys Chem Chem Phys. 2017;19:7228-7235. doi:10.1039/C7CP00540G

22. Yarramala DS, Doshi S, Rao CP. Green synthesis, characterization and antitumor activity of luminescent gold nanoparticles capped with apo- $\alpha$-lactalbumin. $R S C A d v$. 2015;5:32761-32767. doi:10.1039/ C5RA03857J

23. Wen F, Dong Y, Feng L, Wang S, Zhang S, Zhang X. Horseradish peroxidase functionalized fluorescent gold nanodots for hydrogen peroxide sensing. Anal Chem. 2011;83(4):1193-1196. doi:10.1021/ ac1031447

24. Kong Y, Chen J, Gao F, et al. Near-infrared fluorescent ribonuclease-A-encapsulated gold nanoclusters: preparation, characterization, cancer targeting and imaging. Nanoscale. 2013;5(3):1009-1017. doi:10.1039/C2NR32760K 
25. Guével XL, Hötzer B, Jung G, Hollemeyer K, Trouillet V, Schneider M. Formation of fluorescent metal $(\mathrm{Au}, \mathrm{Ag})$ nanodots capped in bovine serum albumin followed by fluorescence and spectroscopy. JPhys Chem C. 2011;115(22):10955-10963. doi:10.1021/jp111820b

26. Yu J, Patel SA, Dickson RM. In vitro and intracellular production of peptide-encapsulated fluorescent silver nanodots. Angew Chem Int Ed. 2007;46(12):2028-2030. doi:10.1002/anie.200604253

27. Granger M, Abadie B, Marchis-mouren G. Limited action of trypsin on porcine pancreatic amylase: characterization of the fragments. FEBS Lett. 1975;56:189-193. doi:10.1016/0014-5793(75)81088-X

28. Yao Y, LiN, Zhang X, et al. DNA-templated silver nanocluster/porphyrin/ $\mathrm{MnO}_{2}$ platform for label-free intracellular $\mathrm{Zn}^{2+}$ imaging and fluorescence-/magnetic resonance imaging-guided photodynamic therapy. ACS Appl Mater Interfaces. 2019;11(15):13991-14003. doi:10.1021/acsami.9b01530

29. Yao Y, Zhao D, Li N, et al. Multifunctional $\mathrm{Fe}_{3} \mathrm{O}_{4} @$ polydopamine@DNA-fueled molecular machine for magnetically targeted intracellular $\mathrm{Zn}^{2+}$ imaging and fluorescence/MRI guided photodynamic-photothermal therapy. Anal Chem. 2019;91(12):7850-7857. doi:10.1021/acs.analchem.9b01591

30. Zhang X, Xi ZQ, Machuki JO, et al. Gold cube-in-cube based oxygen nanogenerator: a theranostic nanoplatform for modulating tumor microenvironment for precise chemo-phototherapy and multimodal imaging. ACS Nano. 2019;13(5):5306-5325. doi:10.1021/acsnano.8b09786
31. Peng Q, Berg K, Moan J, Kongshaug M, Nesland JM. 5-Aminolevulinic acid-based photodynamic therapy: principles and experimental research. Photochem Photobiol. 1997;65(2):235-251. doi:10.1111/php.1997.65.issue-2

32. Peng Q, Warloe T, Berg K, et al. 5-Aminolevulinic acid-based photodynamic therapy: clinical research and future challenges. Cancer. 1997;79(12):2282-2308. doi:10.1002/(SICI)1097-0142(19970615) 79:12<2282::AID-CNCR2 $>3.0 . C O ; 2-\mathrm{O}$

33. Punjabi A, Wu X, Tokatli-apollon A, et al. Amplifying the red-emission of upconverting nanoparticles for biocompatible clinically used prodrug-induced photodynamic therapy. ACS Nano. 2014;8(10):10621-10630. doi:10.1021/nn505051d

34. AI-Kassas R, Donnelly RF, McCarron PA. Aminolevulinic acid-loaded Witepsol microparticles manufactured using a spray congealing procedure: implications for topical photodynamic therapy. $J$ Pharm Pharmacol. 2009;61:1125-1135. doi:10.1211/jpp.61.09. 0001
International Journal of Nanomedicine

\section{Publish your work in this journal}

The International Journal of Nanomedicine is an international, peerreviewed journal focusing on the application of nanotechnology in diagnostics, therapeutics, and drug delivery systems throughout the biomedical field. This journal is indexed on PubMed Central, MedLine, CAS, SciSearch ${ }^{\circledR}$, Current Contents ${ }^{\mathbb{B}} /$ Clinical Medicine,

\section{Dovepress}

Journal Citation Reports/Science Edition, EMBase, Scopus and the Elsevier Bibliographic databases. The manuscript management system is completely online and includes a very quick and fair peer-review system, which is all easy to use. Visit http://www.dovepress.com/ testimonials.php to read real quotes from published authors. 